\title{
FATORES ASSOCIADOS ÀS LIMITAÇÕES FUNCIONAIS EM IDOSAS DE BAIXA RENDA
}

\author{
Jair Sindra Virtuoso Júnior*; Ricardo Oliveira Guerra \\ Trabalho realizado no Programa de Pós-graduação em Ciências da Saúde, Universidade Federal do Rio Grande do Norte, Natal, RN
}

\author{
*Correspondência: \\ Universidade Estadual de \\ Santa Cruz - UESC \\ Departamento de Ciências \\ da Saúde \\ Rodovia llhéus - Itabuna, \\ $\mathrm{Km} 16$ \\ CEP 45662-000 - Ilhéus/ \\ BA - Brasil \\ virtuosoir@yahoo.com.br \\ Tel: (73)3680-5279
}

\begin{abstract}
RESUMO
OBjetivo. Analisar a relação dos aspectos sociodemográficos e referentes às condições de saúde com as limitações funcionais em mulheres idosas de baixa renda do Nordeste brasileiro.

Métodos. Estudo com delineamento transversal, tendo uma amostra representativa de 208 mulheres, com média de 70 anos $( \pm 7,1)$ pertencentes a grupos de convivência e que eram residentes na zona urbana do municíio de Jequié/Bahia. Foi realizada uma bateria de testes físicos, coletadas medidas antropométricas e a aplicação de uma entrevista contendo questões relativas às variáveis sociodemográficas, condições clínicas, saúde percebida, condições físicas e comportamentais. A análise estatística foi realizada com nível de significância $p<0,05$, com cálculo da respectiva razão de chances mediante regressão logística binária, para análise de fatores hierarquicamente agrupados.

Resultados. Encontrou-se uma prevalência de 56\% de mulheres consideradas com limitações funcionais do tipo moderada ou grave, nos quais a partir de análise multivariada hierarquizada, verificou-se associação significativa com a faixa etária superior a 80 anos $(p=0,02)$, condições de viuvez $(p=0,04)$, presença de hipertensão arterial $(p=0,00 \mathrm{I})$, e inatividade física no lazer $(p=0,03)$.

Conclusäo. As características identificadas que se associaram às limitações funcionais sugerem uma complexa rede causal na determinação da condição funcional em mulheres idosas. No entanto, açães direcionadas ao incentivo da prática de atividades físicas no lazer podem contribuir para uma vida com mais qualidade a estas pessoas.
\end{abstract}

UnItermos: Saúde do idoso. Fatores socioeconômicos. Aptidão física.

\section{INTRODUÇ̃̃o}

O declínio da condição funcional com o avanço da idade cronológica é relatado em diversos inquéritos populacionais ${ }^{1,2}$. Em alguns modelos conceituais, fatores específicos de doenças são focalizados como a causa principal das deficiências, predispondo às limitações funcionais $5^{3,4}$.

As limitações funcionais no desempenho das tarefas básicas poderão agir como mediadores primários entre as deficiências e a incapacidade ${ }^{4}$. Morey et al. ${ }^{5}$ acrescentam ainda, a aptidão física como variável de interesse aos modelos teóricos de entendimento do processo de perda da capacidade funcional. Segundo estes autores, parâmetros de aptidão cardiorrespiratória, fatores morfológicos e performance muscular atuariam como percussores de estados patológicos e, ainda, de forma independente, para a exacerbação ou atenuação das limitações funcionais.

A aptidão física expressa a capacidade funcional direcionada à realização da atividade física, representada por um conjunto de componentes que são inatos, adquiridos ou alterados, que se relacionam com a saúde e o desempenho atlético ${ }^{6}$. A capacidade que os idosos têm de manter-se independentes parece depender da manutenção de qualidades físicas relacionadas com a força muscular, flexibilidade, resistência cardiovascular, agilidade e equilíbrio ${ }^{6,7}$. Estes parâmetros físicos definem a base da aptidão funcional, que é caracterizada por Rikli e Jones ${ }^{8}$ como a capacidade fisiológica para executar normal, segura e independente as atividades cotidianas na ausência de fadiga imprópria.

$\mathrm{Na}$ avaliação da aptidão funcional, há necessidade de medidas eficazes que possam ser realizadas com segurança em pessoas idosas?. Apesar da escassez de protocolos avaliativos para populações que apresentam múltiplas limitações, alguns estudos vêm sendo realizados como forma de avaliar o desempenho físico em idosos ${ }^{8,9}$, no sentido de indicar limitações funcionais na realização de atividades espećíficas da vida diária. No entanto, há divergências quanto aos métodos para avaliação das limitações funcionais, sendo ressaltados aspectos positivos e limitações dos métodos de avaliação por testes de auto-percepção em relação aos testes de desempenho físico?.

Há uma carência de estudos que levem em consideração aspectos do desempenho físico (aptidão funcional) conjugados com medidas autopercebidas da condição funcional. E ainda, levantamentos que possam determinar a relação da condição funcional com outros determinantes sociais, demográficos e de saúde em mulheres idosas vivendo em regiões com baixa condição econômica. Em geral, as mulheres idosas quando comparadas aos homens possuem maior chance de serem acometidas por limitações funcionais ${ }^{10}$, sendo que a condição de baixo nível econômico tem sido determinante da perda da condição funcional', ${ }^{\prime}$.

O propósito deste estudo foi analisar a relação das limitações funcionais com as condições sociodemográficas e de saúde em 
mulheres idosas de baixa condição econômica. Os levantamentos de tais informações possibilitam a identificação do status funcional, servindo como referência para fundamentar estratégias de programas direcionados à promoção de saúde, em pessoas idosas de comunidades menos favorecidas economicamente.

\section{Métodos}

Foi realizado um estudo transversal no município de Jequié, localizada 365 km de Salvador, capital da Bahia, Região Nordeste do Brasil, no período de outubro a dezembro de 2006. A amostra inicial de 222 mulheres idosas com 60 anos ou mais foi selecionada de forma probabilística e estratificada por grupo de convivência. O valor da amostra foi proporcional a 42,3\% da população inicial de 528 idosos que representa o número total de sujeitos participantes nos 16 grupos de convivência do município de Jequié, estabelecendo-se nível de confiança de 95\% com erro de 5\%.

Os instrumentos utilizados tiveram sua confiabilidade testada previamente ao estudo. Para tanto foram utilizados a bateria de testes físicos de Fulleton (força de membros inferiores, força de membros superiores, resistência aeróbia, flexibilidade do quadril, agilidade/ equilíbrio dinâmico e índice de massa corporal $)^{8}$ e uma entrevista aplicada de forma individual, para caracterização da amostra com a avaliação de fatores sociodemográficos (idade, educação, classificação socioeconômica ${ }^{12}$ ) e comportamentos relacionados à saúde (saúde percebida, uso de medicamentos, sintomatologia depressival' ${ }^{13}$, atividades instrumentais da vida diária (AIVDs) ${ }^{14}$ e hábitos de prática de atividades físicas no lazer).

Os critérios para inclusão no estudo seguiram o modelo de segurança para a realização de testes físicos em pessoas idosas?. Foram excluídas dos testes as idosas que apresentassem alguma condição médica, limitações físicas ou cognitivas severas que pudesse impedir as instruções de segurança para a realização dos testes, sendo que a pressão arterial era aferida antes e após a realização da bateria de testes; aqueles que apresentassem níveis pressóricos superiores $160 \mathrm{mmHg}$ para a pressão arterial sistólica e $100 \mathrm{mmHg}$ para pressão arterial diastólica foram impedidos de realizar tais testes.

A determinação da presença de limitações funcionais moderada/ grave foi feita na ocorrência de dois resultados concomitantes nas seguintes condições: escore abaixo do percentil 25 para os testes de desempenho motores, índice de massa corporal superior a $24,9 \mathrm{Kg} / \mathrm{m}^{2}$ e escore menor que 13 pontos na escala de autopercepção na avaliação das limitações na realização das atividades instrumentais da vida diária.

Para confecção do banco de dados foi utilizado o software Epidata, versão 3. I b, e as análises por meio do pacote estatíittico SPSS (versão II.0). $\mathrm{Na}$ análise descritiva foi feita a distribuição de freqüências absolutas, a distribuição percentual, média e desvio padrão ( \pm ).

Precedendo a inferência dos dados foram identificados os valores faltosos e, os mesmos ( 14 casos), foram excluídos da planilha de dados. $\mathrm{Na}$ abordagem analítica foi realizada análise de regressão logística univariada ${ }^{15}$. Para a identificação dos fatores associados com a presença de limitações funcionais moderada/grave do idoso foi realizada análise múltipla de regressão logístical', utilizando análise hierarquizada ${ }^{16}$. Mediante a estratégia estabelecida de associações entre as dimensões estudadas (sociodemográfica, condições clínicas e saúde percebida, condições físicas e comportamentais), foram elaborados três modelos explicativos de regressão logística binária, introduzindo as variáveis em forma de blocos, permanecendo no modelo subseqüente apenas aquelas que tiveram significância estatística $(p<0,05)$ no modelo anterior.

O critério de saída para todas as variáveis introduzidas em cada modelo foi $p<0,10$. Ao final, chegou-se a um modelo final de regressão com apenas as variáveis de maior significância estatística. $O$ método adotado para introdução das variáveis nos modelos foi o backward stepwise ${ }^{16}$. Considerou-se um nível de significância $p<0,05$ e intervalo de confiança (IC) de 95\%, com cálculo das razões de chances ajustadas.

Esta pesquisa seguiu os princípios éticos presentes na Declaração de Helsinki e na Resolução n 1 96/96 do Conselho Nacional de Saúde. Os protocolos de pesquisa foram avaliados e aprovados pelo Comitê de Etica em Pesquisa com Seres Humanos da Universidade Estadual do Sudoeste da Bahia (Parecer n⿳ |55/06).

\section{Resultados}

A amostra final do estudo constituiu-se de 208 mulheres idosas, com a média de idade de 70 anos $( \pm 7,1$ ), com a amplitude de 60 a 92 anos. Embora tenham sido observadas algumas variações nos grupos etários estabelecidos, a população em geral pode ser descrita com predominância de viúvas 47,6\% ( $n=99)$, o arranjo familiar caracterizado como sendo multigeracional $34,1 \%(n=7 \mid)$, sendo de baixo nível de escolaridade, em que as que referiram ser analfabetas representavam 38\% $(n=79)$ e com a maioria das participantes classificadas nas classes econômicas "D" e "E", ou seja, baixa classificação socioeconômica 69,2\% ( $n=\mid 46)$.

Alguns comportamentos reportados apresentaram melhoras em relação à freqüência do hábito de vida no presente, como é o caso da ingestão de bebidas alcoólicas em que 32,7\% ( $n=68)$ faziam uso no passado, sendo que apenas 10,1\% ( $n=21)$ mantêm tal hábito, o mesmo acontecendo com o tabagismo, em que foi referido como um hábito do passado por 38\% ( $n=79)$, mas no presente apenas 6,3\% $(n=13)$ permanecem com o uso do tabaco. Já para a prática de atividades físicas foi observado fenômeno inverso, sendo que no passado apenas $18,8 \%(n=39)$ realizavam atividades físicas no momento de lazer e no presente tal hábito foi reportado por $5 \mathrm{I}, 4 \%(n=107)$.

O estado nutricional verificado pelo IMC demonstrou que $62 \%$ $(n=129)$ estavam classificadas na condição de sobrepeso ou obesidade (IMC superior a $\left.24,9 \mathrm{~kg} / \mathrm{m}^{2}\right)$. Os sintomas depressivos foram relatados por $17,3 \%(n=36)$ das participantes, sendo que a alteração do estado cognitivo foi identificada em $74,5 \%(n=155)$. E ainda o uso de medicamentos foi referido por $75,5 \%(n=157)$, sendo que $24 \%(n=50)$ fazem uso de três ou mais medicamentos/dia.

Em relação às doenças auto-referidas, estas foram agrupadas em conformidade a Classificação Internacional de Doenças (CID-10) $)^{17}$, sendo as relativas ao aparelho circulatório $75 \%(n=156)$, sistema osteomuscular 72,6\% $(n=|5|)$, doenças dos olhos $54,3 \%(n=\mid 13)$, ouvidos $41,8 \%(n=87)$ e metabólicas $22,6 \%(n=47)$, respectivamente, as mais referidas pelas idosas entrevistadas. 


\begin{tabular}{|c|c|c|c|}
\hline \multirow[b]{2}{*}{ Percentil } & \multicolumn{3}{|c|}{ Grupo de Idade } \\
\hline & $60-69$ & $70-79$ & $80-92$ \\
\hline Força de membros inferiores (reps) & $(n=109)$ & $(n=83)$ & $(n=20)$ \\
\hline$P_{25}$ & 13,0 & 11,0 & 11,2 \\
\hline$P_{75}^{20}$ & 18,5 & 17,0 & 14,7 \\
\hline Força de membros superiores (reps) & $(n=\mid 10)$ & $(n=83)$ & $(n=20)$ \\
\hline$P_{25}$ & 14,0 & 13,0 & 11,2 \\
\hline$p^{20}$ & 20,0 & 18,0 & 15,7 \\
\hline Resistência aeróbia (nº passos) & $(n=108)$ & $(n=82)$ & $(n=20)$ \\
\hline$P_{25}$ & 79,0 & 72,0 & 59,5 \\
\hline 23 & 106,0 & 98,5 & 89,0 \\
\hline Flexibilidade (cm) & $(n=|| 2)$ & $(n=84)$ & $(n=20)$ \\
\hline$P_{25}$ & 0,0 & 0,0 & $-0,75$ \\
\hline 15 & 9,0 & 6,75 & 8,25 \\
\hline Agglilidade/Equilibrio(seg) & $(n=109)$ & $(n=83)$ & $(n=20)$ \\
\hline$P_{25}$ & 4,8 & 5,5 & 6,2 \\
\hline$P_{75}^{25}$ & 5,8 & 7,0 & 7,6 \\
\hline IMC $\left(\mathrm{Kg} / \mathrm{m}^{2}\right)$ & $(n=|| 5)$ & $(n=85)$ & $(n=22)$ \\
\hline$P_{25}$ & 23,9 & 23,5 & 22,1 \\
\hline$P_{75}^{25}$ & 31,1 & 29,1 & 32,3 \\
\hline
\end{tabular}

Outras condições de saúde foram referidas, a exemplo da ocorrência de quedas nos últimos três meses reportados por $21,2 \%(n=44)$, e de internações nos últimos seis meses $15,4 \%(n=32)$. Há ainda que se ressaltar o fato de que $56 \%(n=122)$ apresentavam limitações funcionais do tipo moderada e/ou grave nas AIVD's. No entanto, a saúde percebida indicou que 34,6\% ( $n=72$ ) possuíam uma percepção otimista da condição de saúde e apenas 17,8\% (n=37) reportaram uma saúde em condição "ruim".

A Tabela I contém os escores percentis $P_{25}$ e $P_{75}$, equivalentes a cada parâmetro da aptidão funcional avaliado (força de membros inferiores e superiores, resistência aeróbia, flexibilidade e agilidade / equilíbrio dinâmico) em três grupos etários (60 - 69 anos, 70 - 79 anos e 80 - 92 anos).

O percentil representa o ponto de localização de uma contagem em relação a uma determinada população. $O$ exemplo que ilustra tal conceito seria o teste de resistência aeróbia, em que o escore de 68 passadas, no grupo etário de 60 a 69 anos, representa que o desempenho do testado se encontra abaixo de $25 \%$ da população com características semelhantes para aquela qualidade física.

Assim, o conhecimento dos escores-percentis para cada parâmetro físico pode auxiliar aos profissionais da área da saúde, na detecção dos componentes da aptidão funcional que necessitam de aprimoramentos.

$\mathrm{Na}$ Tabela 2, estão dispostas as variáveis que se mostraram relacionadas com as limitações funcionais do tipo moderada ou grave. 0 aumento da faixa etária se mostrou com potencial preditivo para o desenvolvimento das limitações funcionais. A condição de viuvez referida pelas entrevistadas em relação as que referiram serem solteiras ou divorciadas foi um fator de risco para a limitação funcional.

\section{Tabela 2 - Análise não ajustada da associação de características sociodemográficas, aspectos clínicos, saúde física e comportamental com as limitaçōes funcionais de mulheres idosas}

\begin{tabular}{|c|c|c|}
\hline Características & Razão de chances [IC95\%] & $\mathrm{p}$ \\
\hline \multicolumn{3}{|l|}{ Sociodemográficas } \\
\hline $\begin{array}{l}\text { Idade (anos) } \\
60-69 \\
70-79 \\
80 \text { ou mais }\end{array}$ & $\begin{array}{c}1,00 \\
1,9[1,04-3,60] \\
9,1[2,03-40,85]\end{array}$ & $\begin{array}{r}0,03 \\
0,001\end{array}$ \\
\hline $\begin{array}{l}\text { Estado civil } \\
\text { Solteira/divorciada } \\
\text { Casada ou vivendo com a parceira } \\
\text { Viúva } \\
\text { Condições clínicas e saúde percebida }\end{array}$ & $\begin{array}{c}1,00 \\
1,2[0,55-2,62] \\
3,2[1,52-6,60]\end{array}$ & $\begin{array}{r}0,63 \\
0,001\end{array}$ \\
\hline $\begin{array}{l}\text { Hipertensão arterial } \\
\text { Ausência } \\
\text { Presençą }\end{array}$ & $\begin{array}{c}1,00 \\
4,2[2,32-7,74]\end{array}$ & 0,001 \\
\hline $\begin{array}{l}\text { Reumatismo } \\
\text { Ausência } \\
\text { Presença }\end{array}$ & $\begin{array}{c}1,00 \\
2,0[1,14-3,66]\end{array}$ & 0,01 \\
\hline $\begin{array}{l}\text { Satisfação com o peso corporal } \\
\text { Sim } \\
\text { Não }\end{array}$ & $\begin{array}{c}1,00 \\
2,0[1,18-3,71]\end{array}$ & 0,01 \\
\hline $\begin{array}{l}\text { Percepção de Saúde } \\
\text { Positiva } \\
\text { Negativa }\end{array}$ & $\begin{array}{c}1,00 \\
2,0[1,14-3,66]\end{array}$ & 0,01 \\
\hline Condições físicas e comportamen & & \\
\hline $\begin{array}{l}\text { Razão cintura/quadril } \\
\leq 0,85 \mathrm{~cm} \\
>0,85 \mathrm{~cm}\end{array}$ & $\begin{array}{c}1,00 \\
2,1[1,05-4,55]\end{array}$ & 0,03 \\
\hline $\begin{array}{l}\text { Quantidade de medicamentos } \\
\text { atéum medicamento } \\
\text { até dois medicamentos } \\
\text { acima de dois medicamentos }\end{array}$ & $\begin{array}{c}1,00 \\
3,7[1,78-7,78] \\
2,8[1,38-5,80]\end{array}$ & $\begin{array}{l}0,001 \\
0,001\end{array}$ \\
\hline $\begin{array}{l}\text { Atividades físicas (presente) } \\
\text { Sim } \\
\text { Não }\end{array}$ & $\begin{array}{c}1,00 \\
2,5[1,46-4,60]\end{array}$ & 0,001 \\
\hline
\end{tabular}

A inatividade física de lazer, no período atual, está relacionada com a limitação funcional, assim como ocorre com a insatisfação corporal e com a medida da relação cintura/quadril superior a $85 \mathrm{~cm}$. O uso de dois ou mais medicamentos contínuos também se mostrou como fator associado às limitações funcionais, do mesmo modo que a percepção negativa de saúde, a presença de hipertensão arterial e de reumatismo.

Os resultados demonstrados na Tabela 2 serviram para determinar a ordenação dos blocos (modelos) e de variáveis a serem inseridas na análise multivariada hierarquizada apresentada na Tabela 3, de modo a explicar melhor as interações entre as características informadas pelas idosas do estudo.

$\mathrm{Na}$ tabela 3, dentre as características sociodemográficas analisadas de forma interagidas no primeiro bloco, apenas a faixa etária e o estado civil se mantiveram relacionados com o declínio da limitação funcional. 
Tabela 3 - Resultados da análise multivariada dos fatores associados às limitações funcionais de mulheres idosas de baixa renda

\begin{tabular}{|c|c|c|c|c|c|c|}
\hline \multirow[b]{2}{*}{ Característica } & \multicolumn{2}{|c|}{ Modelo I } & \multicolumn{2}{|l|}{ Modelo 2} & \multicolumn{2}{|c|}{ Modelo 3} \\
\hline & $\begin{array}{l}\text { Razão de } \\
\text { chances [95\%] }\end{array}$ & $\bar{p}$ & $\begin{array}{l}\text { Razão de } \\
\text { chances [95\%] }\end{array}$ & $\mathrm{p}$ & $\begin{array}{l}\text { Razão de } \\
\text { chances [95\%] }\end{array}$ & $\bar{p}$ \\
\hline \multicolumn{7}{|l|}{$\begin{array}{l}\text { Bloco sóciodemográfico } \\
\text { Idade(anos) }\end{array}$} \\
\hline $\begin{array}{l}60 \text { a } 69 \\
70 \text { a } 79\end{array}$ & $\begin{array}{c}1 \\
1,71[0,90-3,26]\end{array}$ & 0,09 & $\begin{array}{c}1 \\
1,80[0,90-3,597\end{array}$ & 0,09 & $\begin{array}{c}1 \\
1,81[0,90-3,63]\end{array}$ & 0.09 \\
\hline 80 ou mais & $6,28[1,35-29,0]$ & 0,01 & $5,58[1,12-27,78]$ & 0,03 & $5,99[1,21-29,55]$ & 0,02 \\
\hline \multicolumn{7}{|l|}{ Estado civil } \\
\hline Solteira/divorciada & I & & I & & I & \\
\hline Casada ou vivendo com a parceira & $1,23[0,55-2,72]$ & 0,61 & l,80[0,90-3,59] & 0,89 & $0,99[0,41-2,36]$ & 0,99 \\
\hline Viúva & $2,58[1,18-5,60]$ & 0,01 & $5,58[1,12-27,78]$ & 0,04 & $2,37[1,02-5,49]$ & 0,04 \\
\hline \multicolumn{7}{|c|}{$\begin{array}{l}\text { Condições clínicas e saúde percebida* } \\
\text { Hipertensão arterial }\end{array}$} \\
\hline Ausência & & & 1,00 & & 1,00 & \\
\hline $\begin{array}{l}\text { presença } \\
\text { Percepcão de saúde }\end{array}$ & & & $3,57[1,86-6,83]$ & 0,00 & $3,35[1,73-6,47]$ & 0,001 \\
\hline Positiva & & & 1,00 & & 1,00 & \\
\hline Negativa & & & $1,99[1,02-3,87]$ & 0,04 & I,83[0,93-3,59] & 0,08 \\
\hline \multicolumn{7}{|c|}{$\begin{array}{l}\text { Bloco condições físicas e comportamentos** } \\
\text { Práticas de atividades físicas }\end{array}$} \\
\hline $\operatorname{Sim}$ & & & & & 1,00 & \\
\hline Não & & & & & I,99[1,05-3,78] & 0,03 \\
\hline
\end{tabular}

*Ajustado pelo bloco sociodemográico.

***justado pelos blocos sociodemográficos e de condições clínicas e saúde percebida

No segundo bloco analisado, referente às condições clínicas e saúde percebida, de forma controlada para o bloco anterior, a percepção de saúde negativa e a presença de hipertensão arterial permaneceram no modelo.

Já no terceiro bloco, referente as características relativas às condições físicas e comportamentais, apenas a prática de atividades físicas no lazer permaneceu no modelo, sendo que as pessoas que não realizavam práticas de atividades físicas de lazer no presente possuíam aproximadamente três vezes mais chances de ter alguma limitação funcional do tipo moderada ou grave em comparação as outras pessoas que declararam realizar algum tipo de prática de atividade física continuamente no lazer no momento atual.

\section{Discussão}

As limitações funcionais podem ser consideradas como processo que antecede a condiçãao de incapacidade. A identificação de fatores relacionados com as limitações funcionais permite à elaboração de políticas públicas direcionadas à preservação da autonomia do idoso. Nesta discussão abordaremos os principais aspectos encontrados nos nossos resultados, considerando estes como parte de uma cadeia de causalidade determinante das condições que favorecem o aparecimento das limitações funcionais.

A prevalência de limitações funcionais apresentou escore superior a outros levantamentos populacionais ${ }^{2,10}$. O percentual elevado de pessoas com limitações pode ser entendido em parte, considerando as diferenças utilizadas pelos estudos, na construção do indicador para as limitações funcionais. Mas os componentes individuais utilizados como balizadores da condição deficitária de limitações funcionais têm sido destacados na literatura como preditores da condição funcional em pessoas idosas $2,5,7,10$.

Dentre as características sociodemográficas analisadas, somente as variáveis "faixa etária" e "estado civil" se evidenciaram relacionadas com a condição funcional. No geral, é esperado que tenha um declínio das condiçỗes funcionais com o avanço da idade cronológica', 10. E, ainda, que as pessoas na condição de viuvez tenham mais limitações funcionais, do que quando comparado as pessoas solteiras', talvez pelo fato de ter agregado a condições psicológicas de perda familiar e a diminuição da condiçãa financeira. Mas, no presente estudo, não foi identificada uma relaçãao da condição socioeconômica com a funcional. Fato que se justifica pela homogeneidade da população, pois a maior parte vive em condições de pobreza.

As condições clínicas relacionadas com o aparelho circulatório foram as mais prevalentes, sendo que dentre tais problemas a hipertensão arterial se mostrou com uma razão de chances de 4,2 vezes mais para as limitações funcionais. Esse achado corrobora com a literatura, em que a hipertensão arterial e outras enfermidades cardiovasculares são apontadas como fortes fatores de risco para a dependência funcional 1,18 .

A saúde percebida e a satisfação do peso corporal se mantiveram relacionadas com a condição funcional. O entendimento dos aspectos que influenciam a satisfação corporal no idoso é determinante na elaboração de intervenções direcionadas ao bem-estar do mesmo. 
Algumas evidências na literatura demonstram que há um incremento da insatisfação corporal com o aumento dos níveis de sobrepeso e obesidade, principalmente na mulher ${ }^{19,20}$. No entanto, no modelo hierarquizado, a insatisfação com o peso corporal não se manteve como fator associado às limitações funcionais quando adicionada a variável "saúde percebida".

Convém ressaltar que a saúde percebida tem merecido considerável atenção como um indicador do declínio da condição funcional7,2! . uma variável simples de ser obtida, com o potencial de sintetizar uma completa interação de fatores envolvidos na saúde do idoso, e com valor preditivo de mortalidade ${ }^{2 !}$. Considerando que a saúde percebida reflete com uma relativa precisão o estado geral de saúde, as investigações realizadas por Collins et al. ${ }^{21}$ confirmam os achados deste estudo, indicando uma relação positiva entre a saúde percebida e as limitações funcionais. No entanto, não se manteve relacionada às limitações funcionais quando acrescentadas as variáveis físicas e comportamentais.

Nas condições físicas, a razão cintura/quadril superior a $85 \mathrm{~cm}$ representou uma razão de chances de 2,2 vezes mais às limitações funcionais. É válido destacar que além do excesso de peso, a distribuição indesejável da gordura corporal potencializa o risco de doenças cardiovasculares ${ }^{18}$. Há a necessidade de intervenções em comunidades com baixo poder aquisitivo, pois o Nordeste, assim como outras regiões pobres do país, vem passando por um fenômeno denominado de transição nutricional, em que o problema de escassez alimentar tem sido substituído pelo excesso de alimentos de alto teor calórico, que vem gerando um aumento de sobrepeso e obesidade ${ }^{22}$.

Nas variáveis comportamentais, o uso de dois ou mais medicamentos e a ausência de práticas de atividades físicas no lazer se evidenciaram relacionadas com as limitações funcionais. Sendo que no modelo final apenas a atividade física se manteve como fator associado à condição funcional do idoso. Haja vista a inter-relação de níveis elevados de atividade física, com a manutenção dos componentes da aptidão física ${ }^{6}$. Os resultados deste estudo reforçam os achados de Morey et $\mathrm{l}^{5}$, que identificaram efeitos independentes da aptidão física sobre a condição funcional no idoso.

Entre as potenciais e plausíveis limitações deste estudo, poderíamos apontar o próprio delineamento da pesquisa, na qual o fato de ser um estudo transversal nos impedem de assegurar relações implíitas de causalidade entre as variáveis estudadas. No entanto, através de fundamentação encontrada na literatura, no que diz respeito ao grau de associação e das relações causais entre variáveis, nos permite inferir que nossos resultados parecem suportar as evidências dos modelos explicativos de geração das limitações funcionais na população idosa.

A possibilidade de viés de seleção no estudo está minimizada, uma vez que se trata de uma amostra selecionada a partir de uma população homogênea no que diz respeito aos parâmetros socioeconômicos encontrados no Nordeste brasileiro, ou seja, os indicadores sociais indicam baixos estratos de classes economicamente desprovidas. 0 treinamento prévio dos avaliadores para aplicação da entrevista e dos testes de desempenho físico afasta, em grande parte, a possibilidade de viés de aferição. Cabe, no entanto, a possibilidade de que os resultados das medidas auto-relatadas tenham alguma influência de fatores culturais e sociais, como é o caso da baixa escolaridade da amostra estudada.

\section{Conclusão}

Os dados analisados neste estudo permitem concluir que o aumento da idade, a condição de viuvez, a presença de hipertensão arterial, insatisfação com a saúde, a ausência de práticas de atividades físicas de lazer são fatores determinantes para as limitações funcionais das mulheres idosas de baixa renda. Desta forma, a prática de atividade física poderia atuar como um importante fator preditor da condição funcional dessas mulheres, mesmo quando controlado por fatores sociodemográficos, clínicos e comportamentais.

\section{Conflito de interesse: não há}

\section{SUMMARY}

\section{FACTORS ASSOCIATED TO FUNCTIONAL LIMITATIONS IN ELDERLY OF LOW INCOME}

OBIECTIVE. To analyze the relationship of the partner-demographic and relevant aspects to the conditions of health, with the functional limitations in elderly women of low income in the Brazilian Northeast.

METHODS. This cross-sectional study involved a representative sample of 208 women with mean age of 70 years $( \pm 7.1$ ), belonging to coexistence groups and that were resident in the urban zone of the municipal district of Jequié / Bahia. A battery of physical tests was conducted, anthropometric measurements were collected and an interview was made concerning subjects related to partner-demographic variables, clinical conditions and apparent health, physical conditions and behavior. The statistical analysis was carried out with a level of significance of $p<0.05$. Calculation was made of the respective odds ratio by regression binary logistics for analysis of hierarchically clustered factors.

RESULTS. Prevalence offunctional limitations of the moderate or serious type was 56\%. After hierarchical multivariate analysis, significant association was verified with the older age group of 80 years $(p=0.02)$ , conditions of widowhood $(p=0.04)$, presence of arterial hypertension $(p=0.00 I)$, and physical inactivity at leisure $(p=0.03)$.

CONCLUSION. The characteristics identified, associated to functional limitations suggest a complex causal network in the determination of the functional condition of elderly women. However, actions addressed to incentivate practice of physical activities at leisure can contribute to the quality of life of these women. [Rev Assoc Med Bras 2008; 54(5): 430-5]

KEY WORDS: Elderly health. Socioeconomic factors. Physical fitness.

\section{REFERÊNCIAS}

I. Rosa TEC, Benicio MHD, Latorre MRDO, Ramos LR. Fatores determinantes da capacidade funcional entre idosos. Rev Saúde Pública. 2003;37:40-8.

2. Ahecic K, Karehalt T, Thorslund M, Parker MG. Relationships between symptoms, physical capacity and activity limitations in 1992 and 2002. Aging Clin Exp Res. 2007; 19: 1 87-93.

3. Verbrugge LM, Jette A. The disablement process. Soc Sci Med. 1994:38: I- I4.

4. Lawrence RH, Jette AM. Disentangling the disablement process. J Gerontol B Psychol Sci Soc Sci. 1994;5 1: 1 73-82.

5. Morey MC, Pieper CF, Cornoni-Huntley J. Physical fitness and functional limitations in community-dwelling older adults. Med Sci Sports Exerc. 1998;30:715-23. 
6. Shephard RJ, Bouchard C. Principal components of fitness: relationship to physical 'activity and lifestyle. Can J Appl Physiol. 1994; 19:200- 14.

7. Collins K, Rooney BL, Smalley KJ, Havens S. Functional fitness, disease and independence in community-Dwelling older adults in Western Wisconsin. Wisconsin Med J. 2004; 103:42-3.

8. Rikli RE, Jones C]. Functional fitness normative scores for communityresiding older adults, age 60-94. J Aging Phys Act. 1999;7:162-81.

9. Malmberg J, Miilunpalo SI, Vuori IM, Pasanen ME, Oja P, Niemi NAH. A health-related fitness and functional performance test battery for middleaged and older adults: feasibility and health-related content validity. Arch Phys Med Rehabil. 2002;83:666-77.

10. Zunzunegui MV, Nunez O, Durban M, Garcia de Yébones MJ, Otero A. Decreasing prevalence of disability in activities of daily living, functional limitations and poor self-rated health: a 6 year follow-up study in Spain. Age Clin Exp Res. 2006; 1 8:349-5I.

II. Lima-Costa MF, Barreto S, Giatti L, Uchoa E. Desigualdade social e saúde entre idosos brasileiros: um estudo baseado na Pesquisa Nacional por Amostra de Domicílios. Cad Saúde Pública. 2003; 19:745-57.

12. ANEP.Associação Brasileira de Empresas de Pesquisa. Critério de Classificação Econômica Brasil 2003. [citado 20 mar 2003]. Disponível em: hhtp://www.anep.org.br/arquivos/cceb.pdf/.

13. Almeida OP, Almeida SA. Confiabilidade da versão brasileira da escala de depressão em geriatria (GDS) versão reduzida. Arq Neuropsiquiatr. 1999:57:421-6.

14. Lawton MP, Brody EM. Assessment of older people: self-maintaining and instrumental activities of daily living. Gerontologist. 1969;9: 179-86.

15. Hosmer DM, Lemeshow S. Applied logistic regression. New York: John Wiley \& Sons; 1989
16. Victora CG, Huttly SR, Fuchs SC, Olinto MTA. The role of conceptual frameworks in epidemiological analysis: a hierarchical approach. Int J Epidemiol. 1997;26:224-7.

17. Organização Mundial da Saúde. Classificação Internacional de Doenças e problemas relacionados à saúde: décima revisão. São Paulo: Centro Colaborador da OMS para Classificação de Doenças em Português; 1993.

18. Chiba Y, Saitoh S, Takagi S, Ohnishi H, Katoh N, Ohata J, etal. Relationship between viceral fat and cardiovascular disease risk factors: The Tanno and Sobetsu Study. Hypertens Res. 2007;30:229-36.

19. Hrabosky JI, Grilo CM. Body image and eating disordered behavior in a community sample of Black and Hispanic women. Eat Behav. 2007:8: 106- | 4

20. Schwartz MB, Brownell KD. Obesity and body image. Body Image. 2004; : :43-56

21. Nybo H, Petersen HC, Gaist D. Predictors of mortality in 2249 Nonagenarians - the Danish 1905-cohort survey. J Am Geriatr Soc. 2003;51:1365-73.

22. Acuña K, Cruz T. Avaliação do estado nutricional de adultos e idosos e situação nutricional da população brasileira. Arq Bras Endocrinol Metab. 2004:48:345-61.

Artigo recebido: 26/09/07

Aceito para publicação: 15/04/08 